\title{
Cost-effectiveness of a workplace intervention for sick-listed employees with common mental disorders: design of a randomized controlled trial
}

\author{
Sandra H van Oostrom 1,2,3, Johannes R Anema*1,2,3,4, Berend Terluin ${ }^{2,5}$, \\ Henrica CW de Vet ${ }^{2}$, Dirk L Knol ${ }^{2,6}$ and Willem van Mechelen ${ }^{1,2,3,4}$
}

Address: ${ }^{1}$ Department of Public and Occupational Health, VU University Medical Center, Amsterdam, The Netherlands, ${ }^{2}$ EMGO Institute, VU University Medical Center, Amsterdam, the Netherlands, ${ }^{3}$ Body@Work, Research Center Physical Activity, Work and Health, TNO-VU, Amsterdam, The Netherlands, ${ }^{4}$ Research Center for Insurance medicine AMC-UWV-VU University Medical Center, Amsterdam, The Netherlands, ${ }^{5}$ Department of General Practice, VU University Medical Center, Amsterdam, The Netherlands and ${ }^{6}$ Department of Clinical Epidemiology and Biostatistics, VU University Medical Center, The Netherlands

Email: Sandra H van Oostrom - sandra.vanoostrom@vumc.nl; Johannes R Anema* - h.anema@vumc.nl; Berend Terluin - b.terluin@vumc.nl; Henrica CW de Vet - hcw.devet@vumc.nl; Dirk L Knol - d.knol@vumc.nl; Willem van Mechelen - w.vanmechelen@vumc.nl

* Corresponding author

Published: 14 January 2008

BMC Public Health 2008, 8:12 doi:10.1 I86/147I-2458-8-12
Received: 6 December 2007

Accepted: 14 January 2008

This article is available from: http://www.biomedcentral.com/I47I-2458/8/12

(c) 2008 van Oostrom et al; licensee BioMed Central Ltd.

This is an Open Access article distributed under the terms of the Creative Commons Attribution License (http://creativecommons.org/licenses/by/2.0), which permits unrestricted use, distribution, and reproduction in any medium, provided the original work is properly cited.

\begin{abstract}
Background: Considering the high costs of sick leave and the consequences of sick leave for employees, an early return-to-work of employees with mental disorders is very important. Therefore, a workplace intervention is developed based on a successful return-to-work intervention for employees with low back pain. The objective of this paper is to present the design of a randomized controlled trial evaluating the cost-effectiveness of the workplace intervention compared with usual care for sick-listed employees with common mental disorders.

Methods: The study is designed as a randomized controlled trial with a follow-up of one year. Employees eligible for this study are on sick leave for 2 to 8 weeks with common mental disorders. The workplace intervention will be compared with usual care. The workplace intervention is a stepwise approach that aims to reach consensus about a return-to-work plan by active participation and strong commitment of both the sick-listed employee and the supervisor. Outcomes will be assessed at baseline, 3, 6, 9 and 12 months. The primary outcome of this study is lasting return-to-work, which will be acquired from continuous registration systems of the companies after the follow-up. Secondary outcomes are total number of days of sick leave during the follow-up, severity of common mental disorders, coping style, job content, and attitude, social influence, and self-efficacy determinants. Cost-effectiveness will be evaluated from the societal perspective. A process evaluation will also be conducted.
\end{abstract}

Discussion: Return-to-work is difficult to discuss in the workplace for sick-listed employees with mental disorders and their supervisors. Therefore, this intervention offers a unique opportunity for the sick-listed employee and the supervisor to discuss barriers for return-to-work. Results of this study will possibly contribute to improvement of disability management for sick-listed employees with common mental disorders. Results will become available in 2009.

Trial registration: ISRCTN92307/23 


\section{Background} CMDs and sick leave

Common mental disorders (CMDs) are common in the community and often affect functioning to such an extent that they are associated with work absenteeism. In many developed countries, $35 \%$ to $45 \%$ of absenteeism from work is due to mental health problems [1]. Prolonged absence from work often results in a lack of social structure and meaningful activity[2,3] and is associated with a reduced probability of eventual return-to-work (RTW) and an increased probability of subsequent economic and social deprivation $[4,5]$. In the beginning of the sick leave episode the employee is often missed at work and employers will try to facilitate RTW. However, if sick leave continues, the employee will be replaced and the balance will be regained without the sick-listed employee and therefore RTW will become increasingly difficult to accomplish $[6,7]$. Thus, work itself can be an important factor in the RTW process[2]. It is also known that work is a significant contributor to the quality of life [8]. Considering the high costs of sick leave and the adverse consequences of sick leave for employees, an early RTW is very important.

\section{A workplace intervention for CMDs}

A recent publication describes the structured development, implementation and planning for the evaluation of a return-to-work (RTW) intervention for sick-listed employees with common mental disorders (CMDs)[9]. The intervention is based on an existing successful RTW intervention for sick-listed employees with low back pain[10,11]. Until now, for mental health problems the focus of interventions is mainly on the reduction of symptoms, while for musculoskeletal disorders the focus has shifted to the prevention of long-term work disability [12]. The main goal of the intervention for employees with low back pain was the facilitation of RTW[10]. Therefore, employees and their supervisors discussed about barriers for RTW and solutions, and they drew up a plan for implementation of solutions which is based on consensus between the employee and the supervisor.

In the development of such an intervention for sick-listed employees with CMDs the steps of Intervention Mapping were followed[13]. In this, important stakeholders in the process of RTW (i.e. employees recently sick-listed with CMDs, supervisors and occupational health professionals) participated in focus group interviews. Topics like, equality and support in discussions about RTW, the role of an RTW coordinator and the suitable moment to apply this workplace intervention for sick-listed employees were discussed. This resulted in a structured return-to-work intervention, specifically tailored to the needs of sicklisted employees with CMDs [9].

\section{Objective}

The objective of this paper is to describe the design of a cost-effectiveness study of the workplace intervention for sick-listed employees with CMDs. This intervention will be compared with usual care.

\section{Methods}

In order to describe the design of this study we followed the CONSORT statement $[14,15]$, a checklist that intends to improve the quality of reports of randomised controlled trials.

\section{Organisation study}

The study is designed as a randomised controlled trial with a follow-up of one year. The design is presented in Figure 1. Two occupational health services in the Netherlands collaborate in the study, one occupational health service belongs to the VU University and the VU University Medical Center, and the other is attached to CORUS, a steel industry company.

The Medical Ethics Committee of VU University Medical Center approved the study design, protocols, procedures and informed consent. Participation is voluntary and all participants signed informed consent. Towards the involved stakeholders (employees, supervisors and occupational health professionals) the study is entitled the "ADAPT" study.

\section{Participants}

The source population $(n \approx 20.000)$ consists of blue and white collar employees working in the University, the University Medical Center and the steel factory. The source population is diverse: the healthcare sector, the industrial sector and administrative sector are all involved in this study. The workplace intervention is assumed to be appropriate for all these sectors.

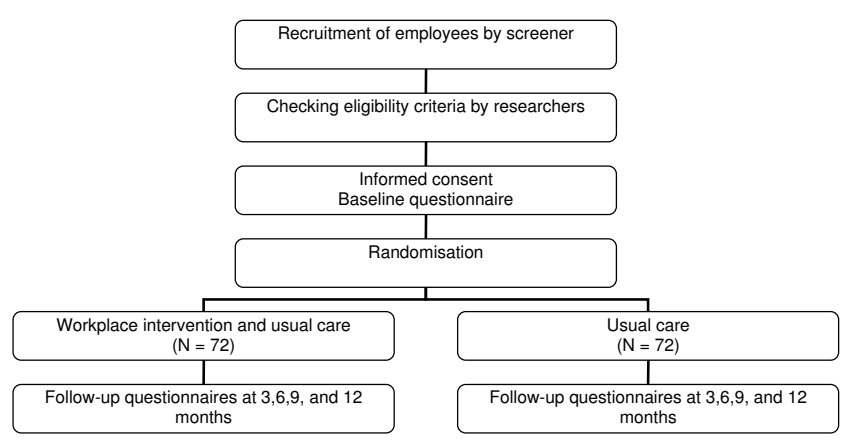

\section{Figure I}

Study design. 
The employees eligible for this study are on sick leave from regular work for 2 to 8 weeks with CMDs. CMDs encompass both criteria-based psychiatric disorders (mostly depressive and anxiety disorders) and 'subthreshold' disorders (including adjustment disorders)[16]. Employees with CMDs are selected on the basis of elevated distress levels and sick leave. Distress reflects the effort people have to put into coping with stressors in order to maintain their habitual level of psychosocial functioning[17]. Severe distress, however, may lead to a breakdown in coping, resulting in psychosocial disfunctioning (e.g. sick leave). It is generally known that distress and depression coexist with chronic diseases and/or physical symptoms [18-21], therefore a heterogeneous group of participants will be selected.

Employees are in the working age range, 18 to 65 years old. Exclusion of employees occurs in case of 1) a conflict between the employee and the employer with legal involvement; 2) working less than 12 hours a week; 3) pregnancy; 4) sick-listed for more than 8 weeks; 5) another episode of sick leave within one month before the current episode; 6) inability to complete questionnaires written in the Dutch language. After randomisation the occupational physician (OP) is responsible to prevent employees with severe psychiatric disorders (mania, psychosis or suicidal) and employees with a terminal illness from starting the workplace intervention.

\section{Recruitment of study population}

All employees sick-listed for more than one week are selected from the databases of the occupational health services and they are sent a letter from their OP with a screening questionnaire. In the letter, the OP requests the employee to fill in the screening questionnaire and to send it back to the researcher. The screening questionnaire contains three questions based on the distress scale of the Four-Dimensional Symptom Questionnaire (4DSQ) $[17,22,23]$ and a question about whether or not the employee is sick-listed. A screening distress score $\geq 4$ corresponds with a 4 DSQ distress score $\geq 11$, the optimal cutoff for any psychosocial problem [23]. The screening procedure allows the researchers to approach the sick-listed employees in an early stage of sick leave, when other treatments have not yet or only just started, and before the 8 weeks RTW plan is started which is obligated according to the Improved Gatekeeper Act.

Employees who return the questionnaire, meet the distress and sick leave criteria and who indicate 'willing to participate' are contacted by the researchers by telephone. In this contact, the researcher provides additional information about the implications of participation and checks the eligibility of the employee by questions about the six exclusion criteria. If an employee meets all the selection criteria and continues to be willing to participate, written information is provided. The researchers plan a face-toface appointment with the employee to give consent, fill in the baseline questionnaire and perform the randomisation.

\section{Usual occupational care in the Netherlands}

The Improved Gatekeeper Act regulates, that the responsibility for RTW is given to the employer and employee together. An employer is obliged to start rehabilitation as soon as possible, in order that the employee can resume own work or other adequate work. The employee should accept the work activities the employer provides. Also, the employee has to visit the OP who can provide advice about RTW and who can guide employees on sick leave with CMDs according to the evidence-based guideline of the Dutch Association of Occupational Physicians (NVAB) published in 2000[7,24]. This guideline aims to provide an optimal functioning of the employee with an $\mathrm{CMD}$ to prevent long-term sick leave and frequent recurrences. The basic idea of this guideline is that recovery can only appear in interaction with the work-environment and should be based on time-contingency. It starts with the establishment of the diagnosis and a listing of the problems within the private life, the work situation and the health care system. The duration of each stage in ordinary recovery is prescribed in the guideline, hence interventions can be initiated when the OP observes that recovery stagnates.

\section{Description of the workplace intervention}

The workplace intervention is based on a cost-effective protocol for sick-listed employees with low back pain $[10,25]$. This protocol is based on principles of 'participatory ergonomics' [26], however applied as a means of tertiary prevention. The process of development of this intervention for employees with CMDs is described elsewhere [9]. The workplace intervention is a stepwise and systematic approach, preceded by a consult with the OP (Figure 2). The objective of the workplace intervention is to reach consensus about an RTW plan by active participation and strong commitment of both the employee and his or her supervisor, guided by an RTW coordinator (in this study a company social worker or a labour expert). The role of the RTW coordinator does not comprise an all knowing expert who advises the employee and the supervisor about the RTW process. The RTW coordinator should provide guidance of the process to reach consensus between the employee and the supervisor about an RTW plan. The employee's and the supervisor's active participation is essential to achieve a sound basis for implementation of the RTW plan. 


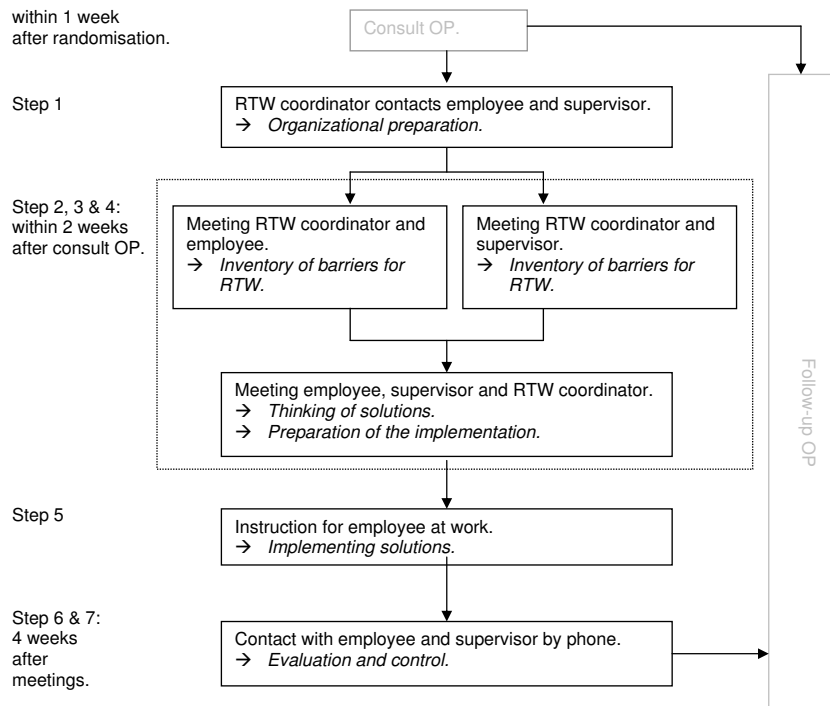

Figure 2

Content of the workplace intervention. The steps of the workplace intervention and the stakeholders involved.

\section{Consult $O P$}

All employees consult the OP before the intervention starts. In this consult the guidelines for usual care are followed. The OP instructs the inventory for stressors to participants as a homework assignment and, if needed, extra consults for stress reduction are planned before the intervention starts. The OP is further responsible to inform the supervisor about the workplace intervention and to ask for his or her participation. To prevent conflicting advise about RTW the OP sends a letter about the workplace intervention and a communication form to the employee's general practitioner. Like in usual care, the OP provides advice about the date of work resumption.

\section{Organizational preparation}

The RTW coordinator contacts the supervisor to check whether the supervisor is sufficiently informed about the intervention and agrees with it. Next, the RTW coordinator informs who is responsible for work adjustments and what procedures should be followed. The employee and supervisor are contacted by phone to plan the three meetings. If required, a checklist with questions about barriers for RTW are sent to the employee and the supervisor by the RTW coordinator. During the meetings with the employee and the supervisor the RTW coordinator emphasizes that the RTW plan does not implicate that the employee is urged to return to work immediately.

\section{Inventory of barriers for RTW}

The meeting of the RTW coordinator and the employee starts with a work visit, to observe the employee's workplace. The elements of work content, work environment, communication and collaboration, and work organization are discussed. This provides the RTW coordinator with a complete picture of the work situation.

Then, the RTW coordinator interviews the employee to obtain a description of the main tasks and specific features of these tasks. For each task, barriers for RTW are summarized and are judged based on the frequency and severity of the barrier. Based on this information, barriers are prioritized in order to select the most important.

Subsequently, a meeting between the RTW coordinator and the supervisor intends to select barriers for RTW of the employee from the supervisors' perspective. This procedure is the same as in the interview between the employee and the RTW coordinator. Then, the RTW coordinator summarizes the results of the two interviews and formulates the barriers to be discussed in the next meeting.

\section{Thinking of solutions}

After the meetings with the employee and the supervisor separately, a meeting takes place with the employee, the supervisor and the RTW coordinator to brainstorm about solutions and to draw up a plan for implementation of solutions. If agreement exist about the barriers to be solved, the RTW coordinator explains the brainstorm procedure. According to the nominal group technique [10] they think of and collect ideas for each solution. All ideas are ordered and judged based on criteria of availability, feasibility and solving capability of the solution and then prioritized. This process is repeated for each barrier. The main goal is to reach consensus between the employee and the supervisor about the most feasible solutions.

\section{Preparation of the implementation}

Together, the employee, the supervisor and the RTW coordinator formulate a plan for implementation of the solutions. This plan describes who is responsible for the implementation of a solution, how this is planned to occur and when the solution should be implemented. The RTW coordinator writes a report about this plan for implementation of solutions and sends it to the employee, the supervisor and the OP.

\section{Implementation of solutions}

In the weeks following the meetings, the solutions are implemented. If required, an RTW coordinator plans a meeting in the workplace to instruct and advise the employee at work. For instance, how to deal with a new job performance or with new equipment. At the same time, the supervisor can be informed about how to 
encourage and guide the employee in his or her (adjusted) work situation.

Evaluation by the RTW coordinator and follow-up by the OP One month after the meetings, the RTW coordinator contacts the employee and the supervisor to inform whether the solutions have been implemented successfully and whether this has contributed to RTW. The RTW coordinator draws up a final report, describing the process and outcome of the implementation and assigns further guidance to the OP.

\section{Training health care professionals}

All OPs involved in this study were trained half a day in the referral of employees to the workplace intervention and the researchers informed them how the workplace intervention is embedded in the guideline for OPs. The referral for the workplace intervention is in line with the guidelines, although explanation about the workplace intervention and standard explanation of the inventory of stressors to the employee is additional. RTW coordinators followed a one-day training course including several roleplayings. Each RTW coordinator who guides a first case according to the protocol was contacted by the researchers to facilitate the process. Two follow-up training sessions were conducted during the recruitment period to discuss difficulties and to practise with cases.

\section{Sample size}

Time to lasting RTW is the primary outcome measure for the power calculation. In order to calculate the sample size we assumed that a Hazard Ratio (HR) of 2.0 is the smallest clinical and societal relevant ratio. A HR of 2.0 indicates that employees in the intervention group return to work twice as quickly as employees in the control group. This HR is based on recent studies in occupational health care on RTW of short-term sick-listed employees with low back pain and adjustment disorders $[11,25,27,28]$. Assuming that a minimum of $2 / 3$ of the participants achieve a full RTW during the follow-up, the calculation showed that a sample size of 98 employees is needed (a power of $(1-\beta=) 0.80$ and a two-sided significance level of $0.05(\alpha))$. Since the OPs, because of their role and function in the RTW process, may influence the RTW date, a multilevel analysis on the level of the OP is taken into account. For 13 OPs and an intraclass correlation coefficient (ICC) of 0.05 (clustering in our groups is not presumed to be large) a total of 130 employees are needed. When taking into account a loss to follow-up of $10 \%, 144$ employees have to be enrolled in the study. Although loss to follow-up is taken into account, we do not expect a high loss to follow-up in this study because data about the primary outcome (lasting RTW) will be acquired from the continuous company registration systems after the one-year follow-up.

\section{Randomisation procedure}

An independent statistician prepared the randomisation by using a computer-generated randomisation. To prevent unequal randomisation, employees are pre-stratified by company and whether they are on full or part-time sick leave. Furthermore, block randomisation (with blocks of four) is applied to ensure equal group sizes within each stratum. The researchers prepared sealed envelopes before the start of the study containing either a referral to the workplace intervention group or the usual care group. If the baseline questionnaire is completed, each employee can choose one of the two succeeding envelopes of the correct stratum provided by the researcher. The employee is asked to open the envelope and write down his or her name and the date on the note that contains the randomisation result.

\section{Blinding}

The participants, the occupational health professionals and the researcher are not blinded for the group assignment. It is likely that several participants of one department will participate and therefore knowledge about both groups is provided for the participants. Furthermore, OPs will be visited by both employees in the workplace intervention group and employees in the usual care group. Therefore they cannot be blinded. Since all follow-up questionnaires are sent to the employee by mail, no direct influence by the researchers or occupational health professionals is likely to occur.

The registration of sick leave in the Netherlands is done by companies and managed by the occupational health services. Since these measurements are extracted from computerized databases, bias caused by a lack of blinding is prevented for this outcome. Since the secondary outcomes are all self-reported, blinding is impossible. After randomisation all participants receive a research code consisting of a consecutive number. A research assistant will put all data in the computer by the research code. Therefore, the analysis of the data by the researcher will be blind.

\section{Co-interventions and compliance}

Co-interventions can not be avoided, because less employees will participate when asking to stop with or stay away from other treatments. In both the intervention and control groups co-interventions are assessed in each follow-up measurement. The data about co-interventions on baseline can be used to adjust for co-interventions in the final multivariate analyses if necessary. In the intervention group the compliance to the workplace intervention will be measured by asking employees, supervisors, OPs and RTW coordinators independently about the intervention applied. 


\section{Contamination}

As randomisation is performed at the level of the employee, OPs who are trained in the workplace intervention guide participants in both the workplace intervention group and the usual care group. However, the actual intervention will be applied by RTW coordinators. They are asked to avoid application of components of the workplace intervention in case of guidance of employees who are in the usual care group. To prevent contamination and role confusion, RTW coordinators will not apply the workplace intervention for employees from departments they work for regularly.

\section{Outcomes}

This study has a one-year follow-up with measurements scheduled at 3, 6, 9, and 12 months after baseline. After the baseline measurement, all questionnaires are sent to the employees by mail. Data on absenteeism are registered continuously by the companies and will be acquired from the registration systems after the one-year follow-up. These data will be checked with information in the medical file of each employee in the occupational health services. If the data are not consistent, the OP will be asked for clarification. Data about the diagnoses will be obtained from the medical file of each employee. OPs in the Netherlands classify diagnoses according to the Classification of diseases (CAS)[29] which is based on the ICD-10.

\section{Effect evaluation}

The primary outcome measure in this study is lasting RTW, defined as: duration of sick leave with CMDs in calendar days from the day of randomization until full RTW in own or other work with equal earnings, for at least 4 weeks without (partial or full) recurrence. This means that recurrences of sick leave within 4 weeks of full RTW are considered as belonging to the preceding period of sick leave.

Secondary outcome measures are:

- Total number of days of sick leave. These will be calculated for the entire follow-up period.

- Severity of CMD symptoms. Changes in symptoms of distress, depression, anxiety, and somatization are measured by the Four-Dimensional Symptom Questionnaire (4DSQ). The 4DSQ is a valid self-report questionnaire to measure distress, depression, anxiety and somatization in a working population $[17,23]$.

- Coping style. This outcome is measured with the Ways of Coping Questionnaire (WCQ) [30], which is based on Lazarus' Theory of Stress and Coping[31]. Only two dimensions of the WCQ are included. These dimensions are avoidance and planful problem-solving, to evaluate whether the workplace intervention influences these ways of coping.

- Job content. The Job Content Questionnaire (JCQ)[32] is used to measure job content at baseline and 3 months. Job content data can either be prognostic or provide insight in working mechanism of the workplace intervention.

- Attitude, Social influence and self-Efficacy (ASE) determinants. The ASE-model could provide insight into the working mechanism of the workplace intervention [9]. Questions about attitude, social influence, self-efficacy, and barriers and facilitators were formulated, based on a validated structure of the questions often used in health promotion research[33,34] and are incorporated in the questionnaire at baseline and after 3 months. The questions are measured on bipolar five-point Likert scales.

\section{Prognostic measures}

Sick leave in the past year, burnout and expectations of the employee about the duration of absence[35] are considered to be potentially prognostic for RTW. Burnout is measured by the Utrecht Burnout Scale-General Survey UBOS[36], the Dutch version of the Maslach Burnout Inventory [37]. In addition, the amount of psychosocial problems may be a prognostic factor for CMDs and sick leave. Psychosocial problems over the last 6 months are measured by the BIOPRO questionnaire [38].

\section{Cost-effectiveness evaluation}

Cost-effectiveness will be evaluated from the societal perspective. Direct costs of health care usage are measured by the Tic-P questionnaire [39]. The Tic-P is developed for medical costs relevant to the treatment of mental health disorders, such as visits to general practitioner, occupational physician, psychologist, psychiatrist, social worker, admission into a hospital, use of medication etc. Health care costs will be valued according to the prices suggested in the guidelines for economic evaluation in The Netherlands [40]. If cost-guidelines are not available, costs will be estimated using real prices or population-based estimates if available in the literature. Costs of lost productivity caused by (partial) sick leave due to CMDs are calculated from the number of days of sick leave and lost earnings, as provided by the occupational health services. Indirect costs can be calculated using the friction cost approach and the human capital approach, based on income as provided by the employee or as derived from function, age and gender. To compare the results of the cost effectiveness analysis with other conditions, general health status is measured according to the standard Dutch version of the EuroQol EQ-5D[41]. 


\section{Process evaluation}

A process evaluation is conducted for the first 35 employees who actually received the workplace intervention. A questionnaire is sent to their supervisor, the OP and the RTW coordinator as well. For employees, the questionnaire is included in the postal questionnaire after 3 months and contains questions about employee satisfaction, the work adaptations chosen, the expected effect of work adaptations, and the compliance with workplace intervention process. Employee satisfaction is measured with the short version of the Patient Satisfaction with Occupational Health Services Questionnaire (PSOHQ)[42]. In addition, the barriers for RTW, the solutions and the RTW plan discussed in the meetings are collected within standardized schemes. All identified barriers for RTW and solutions will be analyzed qualitatively and classified by two researchers independently. The classification will be based on a simplified version of the 'Ergonomic Abstracts' classification scheme $[43,44]$.

\section{Statistical analyses}

All statistical analyses will be performed at employee level, according to the intention-to-treat principle. In order to assess whether protocol deviations have caused bias, the results of the intention-to-treat analyses will be compared to per protocol analyses, excluding those employees who were not treated according to the intervention protocol. Baseline characteristics of employees in the two groups will be compared using descriptive statistics. If necessary, analyses will be adjusted for prognostic dissimilarities.

\section{Effect evaluation}

Survival analysis will be used to analyse sick leave data with regard to the first period of sick leave. To describe the sick leave duration until lasting RTW in both groups, the Kaplan Meier method will be used. The Cox proportional hazard model will be applied to calculate hazard ratios. If appropriate standard errors will be corrected for clustering. Differences in total days of sick leave during the year of follow-up will be analysed by using the Student's T-test. Longitudinal random coefficient analyses will be used to assess differences in secondary outcome measures. Intraclass correlation coefficients will be calculated.

\section{Cost-effectiveness evaluation}

Direct, indirect and total costs will be computed for each employee. Bootstrapping will be used for pair-wise comparison of the mean groups to calculate mean differences in direct, indirect and total costs between the two groups of employees. Confidence intervals (95\%) will be obtained by bias corrected and accelerated bootstrapping. To assess the cost-effectiveness ratios of the workplace intervention, the difference in mean costs between the groups will be divided by the difference in RTW between the groups. These ratios will be graphically presented in a cost-effectiveness plane. Acceptability curves will also be presented. Similarly, utility assessed with the EuroQol EQ-5D will be used to estimate the incremental costs per QALY gained in a cost-utility analysis.

\section{Discussion}

This study protocol presents a randomised controlled trial to investigate the cost-effectiveness and feasibility of the workplace intervention for sick-listed employees with CMDs. This intervention offers an unique opportunity for the sick-listed employee and his or her supervisor to discuss barriers for RTW related to physical and mental workload. Especially for sick leave due to CMDs, RTW is difficult to discuss in the workplace $[45,46]$. The screening procedure selects employees who are sick-listed with CMDs. This means that we select not only employees with mental disorders solely, but also employees with a combination of physical and mental complaints. However, application of this intervention and generalizing the results directly to other countries will be difficult because the workplace intervention should always be tailored to the social, political and cultural context $[9,47]$.

\section{Methodological considerations}

A limitation of this study is that it is not possible to blind the employees and health care providers for the intervention allocation, because of the nature of the workplace intervention. The data of the primary outcome will be extracted from databases, therefore no bias will be introduced due to non-blinded employees or OPs for this outcome. A strength of this study is that owing to the screening procedure selection bias is restricted, because the OP has no role in the inclusion of participants. However, selection bias may occur due to self-selection of employees.

There is increasing interest in RTW as an outcome in research. However, little agreement exists about the source of the RTW data. A workers compensation database might underestimate sick-listed days compared with self report by the employee $[48,49]$. Also, several definitions of RTW are available in the occupational health field [50]. The definition of lasting RTW that we use is the most strictest known and could result in the lowest percentage RTW. For a successful RTW it takes into account not only speed but also durability of RTW. However, in a study that compared several RTW definitions, almost the same magnitude of effect of the interventions was found, regardless of the definition used [51].

Furthermore, we are limited to a one-year follow-up of the sick-listed employees with CMDs. From an economic perspective, a longer follow-up is preferable to investigate intervention effects over several years. Also, productivity 
loss prior and after the episode of sick leave due to CMDs, is not measured in this study. Even if an employee is not absent from work, CMDs can cause a substantial reduction in productivity [52]. For this study, no information can be generated about differences in productivity losses between the workplace intervention group and the usual care group. Brouwer et al. estimated that productivity loss both prior and after the episode of sick leave can lead to an increase in estimated production losses of about 14\% [53].

\section{Impact of results}

The results of this study will possibly contribute to treatment options in occupational health practice, for sicklisted employees with CMDs. In the Netherlands the workplace intervention has already proved to be effective for low back pain. Positive results for sick-listed employees with CMDs could offer extended possibilities for implementation of the workplace intervention in usual care. Occupational health professionals as well as supervisors and employees can possibly benefit from a structured approach to identify and discuss barriers for RTW and the development of an consensus-based RTW plan. Results of this study will become available in 2009.

\author{
Abbreviations \\ ASE = Attitude-Social influence-self-Efficacy \\ JCQ = Job Content Questionnaire \\ $\mathrm{CMD}=$ common mental disorder \\ $\mathrm{OP}=$ occupational physician \\ RTW = return-to-work \\ UBOS = Utrecht Burnout Scale \\ WCQ = Ways of Coping Questionnaire
}

PSOHQ = Patient Satisfaction with Occupational Health Services Questionnaire

4DSQ = Four-Dimensional Symptom Questionnaire

\section{Competing interests}

The author(s) declare that they have no competing interests.

\section{Authors' contributions}

SHO is responsible for the data collection and drafted the manuscript. SHO and JRA developed the study design. JRA was the main applicant and BT and WM were coapplicants on the successful funding proposal. All authors participated in discussing the design of the study and developing the intervention protocols. All authors have read and corrected draft versions of the manuscript and approved the final manuscript.

\section{Acknowledgements}

This study is financially supported by the Dutch Ministry of Social Affairs and Employment and the participating occupational health services.

\section{References}

I. NKAP (Dutch Knowledge Centre of Work and Mind: Factsheet 2, facts and figures about work-related psychological disorders. [In Dutch: Factsheet 2, feiten en cijfers over arbeidsrelevante psychische aandoeningen]. Utrecht 2004.

2. Department of Health: Choosing health: making healthier choices easier. (Public Health White Paper) London: DH; 2004.

3. The Dutch Institute of Psychologists (NIP) and the Dutch Association of Primary Care Psychologists (LVE): Work and mental health problems. Explanation about guidelines for psychologists Amsterdam: NIP/LVE; 2005.

4. Henderson M, Glozier N, Holland EK: Long term sickness absence is caused by common conditions and needs managing. $B M J$ 2005, 330:802-803.

5. Hensing G, Brage S, Nygard JF, Sandanger I, Tellnes G: Sickness absence with psychiatric disorders - an increased risk for marginalisation among men? Soc Psychiatry Psychiatr Epidemiol 2000, 35:335-340.

6. Commissie Psychische Arbeidsongeschiktheid [Committee for Occupational Disability due to mental health problems]: Leidraad Aanpak verzuim om psychische redenen [Guidelines for Approaching Absenteeism due to Mental Health Problems]. Commissie Psychische Arbeidsongeschiktheid 200I.

7. van der Klink JJ, ed: Guidelines for Mental Health Problems Eindhoven: NVAB (Dutch Association of Occupational Physicians); 2000.

8. Bowling A: What things are important in people's lives? A survey of the public's judgements to inform scales of health related quality of life. Soc Sci Med 1995, 4I:| 1447- I462.

9. van Oostrom SH, Anema JR, Terluin B, Venema A, de Vet HC, van Mechelen W: Development of a workplace intervention for sick-listed employees with stress-related mental disorders: Intervention Mapping as a useful tool. BMC Health Serv Res 2007, 7:127.

10. Anema IR, Steenstra IA, Urlings IJ, Bongers PM, de Vroome EM, van Mechelen W: Participatory ergonomics as a return-to-work intervention: a future challenge? Am J Ind Med 2003, 44:273-28I.

II. Anema JR, Steenstra IA, Bongers PM, de Vet HCW, Knol DL, van Mechelen W: Multidisciplinary Rehabilitation for Subacute Low Back Pain: Graded Activity or Workplace Intervention or Both? A Randomized Controlled Trial. Spine 2007, 32:291-298.

12. Loisel P, Durand MJ, Berthelette D, Vezina N, Baril R, Gagnon D, Lariviere C, Tremblay C: Disability prevention - New paradigm for the management of occupational back pain. Dis Manage Health Outcomes 200I, 9:35I-360.

13. Bartholomew LK, Parcel GS, Kok GJ, Gottlieb NH: Intervention Mapping: designing theory and evidence-based health promotion programs Mountain View, California: Mayfield Publishing Company; 200I.

14. Altman DG, Schulz KF, Moher D, Egger M, Davidoff F, Elbourne D, Gotzsche PC, Lang T: The revised CONSORT statement for reporting randomized trials: explanation and elaboration. Ann Intern Med 200I, 134:663-694.

15. Begg C, Cho M, Eastwood S, Horton R, Moher D, Olkin I, Pitkin R, Rennie D, Schulz KF, Simel D, Stroup DF: Improving the quality of reporting of randomized controlled trials. The CONSORT statement. JAMA 1996, 276:637-639.

16. Glozier N: Mental ill health and fitness for work. Occup Environ Med 2002, 59:714-720.

17. Terluin B, van Rhenen W, Schaufeli WB, de Haan M: The FourDimensional Symptom Questionnaire (4DSQ): measuring distress and other mental health problems in a working population. Work Stress 2004, I 8: 187-207.

18. Gatchel RJ: Comorbidity of chronic pain and mental health disorders: the biopsychosocial perspective. Am Psychol 2004, 59:795-805. 
19. Dersh J, Gatchel RJ, Polatin P, Mayer T: Prevalence of psychiatric disorders in patients with chronic work-related musculoskeletal pain disability. J Occup Environ Med 2002, 44:459-468.

20. Lotters F, Franche RL, Hogg-Johnson S, Burdorf A, Pole JD: The prognostic value of depressive symptoms, fear-avoidance, and self-efficacy for duration of lost-time benefits in workers with musculoskeletal disorders. Occup Environ Med 2006, 63:794-801.

21. Haldorsen EM, Kronholm K, Skouen JS, Ursin H: Multimodal cognitive behavioral treatment of patients sicklisted for musculoskeletal pain: a randomized controlled study. Scand J Rheumatol 1998, 27:16-25.

22. Bakker IM, Terluin B, van Marwijk HW, Gundy CM, Smit JH, vanMechelen W, Stalman WA: 'Effectiveness of a Minimal Intervention for Stress-related mental disorders with Sick leave (MISS); study protocol of a cluster randomised controlled trial in general practice [ISRCTN4377964I]. BMC Public Health 2006, 6:124.

23. Terluin B, van Marwijk HW, Ader HJ, de Vet HC, Penninx BW, Hermens ML, van Boeijen CA, van Balkom AJ, van der Klink J], Stalman WA: The Four-Dimensional Symptom Questionnaire (4DSQ): a validation study of a multidimensional self-report questionnaire to assess distress, depression, anxiety and somatization. BMC Psychiatry 2006, 6:34

24. van der Klink JJ, van Dijk FJ: Dutch practice guidelines for managing adjustment disorders in occupational and primary health care. Scand J Work Environ Health 2003, 29:478-487.

25. Loisel P, Abenhaim L, Durand P, Esdaile JM, Suissa S, Gosselin L, Simard R, Turcotte J, Lemaire J: A population-based, randomized clinical trial on back pain management. Spine 1997, 22:291।-2918

26. de Jong AM, Vink P: Participatory ergonomics applied in installation work. Appl Ergon 2002, 33:439-448.

27. Staal JB, Hlobil H, Twisk JW, Smid T, Koke AJ, van Mechelen W: Graded activity for low back pain in occupational health care: a randomized, controlled trial. Ann Intern Med 2004, 140:77-84

28. van der Klink J, Blonk RW, Schene AH, van Dijk FJ: Reducing long term sickness absence by an activating intervention in adjustment disorders: a cluster randomised controlled design. Occup Environ Med 2003, 60:429-437.

29. Lisv: CAS: Classification of symptoms, diseases and causes for occupational and insurance physicians Utrecht: Lisv; 1997.

30. Bramsen I, Bleiker EMA, Tiemstra AHM, Rossum SMGv, van der Ploeg HM: A Dutch adaptation of the ways of coping questionnaire: factor structure and psychometric properties. Anxiety Stress Coping 1995, 8:352.

31. Lazarus RS, Folkman S: Stress, appraisal and coping New York: Springer Publishing Company; 1984.

32. Karasek R, Brisson C, Kawakami N, Houtman ILD, Bongers PM, Amick BC: The Job Content Questionnaire (JCQ): an instrument for internationally comparative assessments of psychosocial job characteristics. J Occup Health Psychol 1998, 3:322-355

33. Damoiseaux V, van der Molen HT, Kok GJ: Gezondheidsvoorlichting en gedragsverandering 3rd edition. Assen, Heerlen: Van Gorcum, Open Universiteit; 1998.

34. de Vries H, Dijkstra M, Kuhlman P: Self-efficacy: The third factor besides attitude and subjective norm as a predictor of behavioural intentions. Health Educ Res 1988, 3:273-282.

35. Nieuwenhuijsen K, Verbeek JH, de Boer AG, Blonk RW, van Dijk FJ: Predicting the duration of sickness absence for patients with common mental disorders in occupational health care. Scand J Work Environ Health 2006, 32:67-74.

36. Schaufeli WB, van Dierendonck D: Utrecht Burnout Scale: Manual (UBOS 2000). Swets Test Publishers (STP); 2000.

37. Maslach C, Jackson SE: Maslach Burnout Inventory: manual research edition Palo Alto: CA: Consulting Psychologists Press; 1986.

38. Hosman CMH: Psychosocial problems and seeking assistence. A social epidemiological study on behalf of preventive mental health care 1983.

39. Hakkart-van Roijen L: Manual Trimbos/iMTA questionnaire for Costs associated with Psychiatric Illness (TiC-P). Institute for Medical Technology Assessment 2002.

40. Oostenbrink JB, Bouwmans CAM, Koopmanschap MA, Rutten FFH: Manual for cost studies, methods and standard cost-prices for economic evaluation in health care. [In Dutch: Handleiding voor kostenonderzoek, methoden en standaard kostprijzen voor economische evaluaties] Board of health care insurance; 2004.

41. Dolan P: Modeling valuations for EuroQol health states. Med Care 1997, 35: 1095-I 108.

42. Verbeek JH, de Boer AG, van der Weide WE, Piirainen H, Anema JR, van Amstel RJ, Hartog F: Patient satisfaction with occupational health physicians, development of a questionnaire. Occup Environ Med 2005, 62:1 19-123.

43. Stapleton C: Classification scheme. In Ergonomics Abstracts Vol London: Taylor \& Francis Ltd; 2000:i-vii.

44. National Institute for Occupational Safety and Health: National occupational research agenda (NORA) Cincinnati: $\mathrm{OH}$ : US Department of Health and Human Services; 1996.

45. Jettinghoff K, Venema A, Anema JR, Steenstra IA: Toepasbaarheid van de Participatieve Aanpak Werkaanpassing bij verzuim ten gevolge van psychische werkbelasting (Feasability of the Participative Approach to Work Adjustment in Sick Leave caused by Psychological Job Strain). TBV 2005, I 3:294-299.

46. Stuart H: Stigma and work. Healthc Pap 2004, 5: I 00- I I I.

47. Goldenhar LM, LaMontagne AD, Katz T, Heaney C, Landsbergis P. The intervention research process in occupational safety and health: an overview from the National Occupational Research Agenda Intervention Effectiveness Research team. J Occup Environ Med 2001, 43:6 I6-622.

48. Dasinger LK, Krause N, Deegan LJ, Brand RJ, Rudolph L: Duration of work disability after low back injury: a comparison of administrative and self-reported outcomes. Am J Ind Med I999, 35:619-631.

49. Pole JD, Franche RL, Hogg-Johnson S, Vidmar M, Krause N: Duration of work disability: a comparison of self-report and administrative data. Am J Ind Med 2006, 49:394-40I.

50. de Vet HCW, Heymans MW, Dunn KM, Pope DP, van der Beek AJ, Macfarlane GJ, Bouter LM, Croft PR: Episodes of low back pain: a proposal for uniform definitions to be used in research. Spine 2002, 27:2409-24l6.

5I. Steenstra IA, de Vroome EM, de Vet HCW, Bongers PM, van Mechelen W: Defining return to work, examples from a trial. Palmo International Forum IX: Primary care research on low back pain; Palma de Mallorca .

52. World Health Organization: Mental health policies and programmes in the workplace. Mental health policy and service guidance package 2005.

53. Brouwer WB, van Exel NJ, Koopmanschap MA, Rutten FF: Productivity costs before and after absence from work: as important as common? Health Policy 2002, 61 : I73-187.

\section{Pre-publication history}

The pre-publication history for this paper can be accessed here:

\section{http://www.biomedcentral.com/1471-2458/8/12/prepub}

Publish with Bio Med Central and every scientist can read your work free of charge

"BioMed Central will be the most significant development for disseminating the results of biomedical research in our lifetime. "

Sir Paul Nurse, Cancer Research UK

Your research papers will be:

- available free of charge to the entire biomedical community

- peer reviewed and published immediately upon acceptance

- cited in PubMed and archived on PubMed Central

- yours - you keep the copyright 